\title{
Solar-panel and parasol strategies shape the proteorhodopsin distribution pattern in marine Flavobacteriia
}

\author{
Yohei Kumagai ${ }^{1,2}$. Susumu Yoshizawa $\oplus^{1,2} \cdot$ Yu Nakajima ${ }^{1,2} \cdot$ Mai Watanabe ${ }^{3} \cdot$ Tsukasa Fukunaga $^{4} \cdot$ \\ Yoshitoshi Ogura $^{5}$. Tetsuya Hayashi ${ }^{5} \cdot$ Kenshiro Oshima $^{6} \cdot$ Masahira Hattori $^{6,7} \cdot$ Masahiko Ikeuchi $^{3}$. \\ Kazuhiro Kogure ${ }^{1,2} \cdot$ Edward F. DeLong ${ }^{8} \cdot$ Wataru Iwasaki $\mathbb{D}^{1,4,9}$
}

Received: 29 September 2017 / Revised: 17 December 2017 / Accepted: 2 January 2018 / Published online: 6 February 2018

(c) The Author(s) 2018. This article is published with open access

\begin{abstract}
Proteorhodopsin (PR) is a light-driven proton pump that is found in diverse bacteria and archaea species, and is widespread in marine microbial ecosystems. To date, many studies have suggested the advantage of PR for microorganisms in sunlit environments. The ecophysiological significance of PR is still not fully understood however, including the drivers of PR gene gain, retention, and loss in different marine microbial species. To explore this question we sequenced 21 marine Flavobacteriia genomes of polyphyletic origin, which encompassed both PR-possessing as well as PR-lacking strains. Here, we show that the possession or alternatively the lack of PR genes reflects one of two fundamental adaptive strategies in marine bacteria. Specifically, while PR-possessing bacteria utilize light energy ("solar-panel strategy"), PR-lacking bacteria exclusively possess UV-screening pigment synthesis genes to avoid UV damage and would adapt to microaerobic environment ("parasol strategy"), which also helps explain why PR-possessing bacteria have smaller genomes than those of PR-lacking bacteria. Collectively, our results highlight the different strategies of dealing with light, DNA repair, and oxygen availability that relate to the presence or absence of PR phototrophy.
\end{abstract}

\section{Introduction}

Proteorhodopsin (PR) plays a fundamental role in marine ecosystems as a light-driven proton pump protein that converts light energy to proton motive force [1]. While this

Electronic supplementary material The online version of this article (https://doi.org/10.1038/s41396-018-0058-4) contains supplementary material, which is available to authorized users.

Susumu Yoshizawa

yoshizawa@aori.u-tokyo.ac.jp

$\triangle$ Wataru Iwasaki

iwasaki@bs.s.u-tokyo.ac.jp

1 Atmosphere and Ocean Research Institute, The University of Tokyo, Chiba 277-8564, Japan

2 Department of Natural Environmental Studies, Graduate School of Frontier Sciences, The University of Tokyo, Chiba 277-8563, Japan

3 Department of Life Sciences, Graduate School of Arts and Sciences, The University of Tokyo, Tokyo 153-8902, Japan

4 Department of Computational Biology and Medical Sciences, proton motive force is sufficient to generate ATP $[2,3]$, PRpossessing $(\mathrm{PR}+)$ prokaryotes may also utilize light energy to uptake organic compounds [4]. Pioneering studies on PR + marine Flavobacteriia showed that light fosters bacterial growth [5] and that their PR expression is likely coupled with carbon assimilation through anaplerotic inorganic carbon fixation [6, 7]. PR-mediated photoheterotrophy is broadly distributed among various groups of marine prokaryotes in which Alphaproteobacteria,

Graduate School of Frontier Sciences, The University of Tokyo, Chiba 277-8561, Japan

5 Department of Bacteriology, Faculty of Medical Sciences, Kyushu University, Fukuoka 812-8582, Japan

6 Center for Omics and Bioinformatics, Graduate School of Frontier Sciences, The University of Tokyo, Chiba 277-8561, Japan

7 Graduate School of Advanced Science and Engineering, Waseda University, Tokyo 169-8555, Japan

8 Center for Microbial Oceanography: Research and Education, University of Hawaii, Honolulu, HI 96822, USA

9 Department of Biological Sciences, Graduate School of Science, The University of Tokyo, Tokyo 113-0032, Japan 
Gammaproteobacteria, and Flavobacteriia are the major groups, being consistent with the estimated physiological benefits of having PR. Recent culture-independent surveys showed that PR genes can occur in up to $80 \%$ of prokaryotes in the marine photic zone [8], and their RNA and protein expression levels are high [4, 9]. Overall, accumulating evidence suggests that possessing PR is generally advantageous to an organism in sunlit marine microbial ecosystems.

On the other hand, the growing understanding of PR function provokes another fundamental question-if the possession of $\mathrm{PR}$ is so advantageous acting as bonus "solar panels" for microbes, why are there so many PR-lacking $(\mathrm{PR}-)$ prokaryotes in the marine photic zone [3]. Comparative genomics is a potentially useful approach for answering such questions because genomes fundamentally reflect microbial ecophysiology [10-13]. That is, systematic differences between PR - and PR + prokaryote genomes might provide clues about differences in the lifestyles of these microbes. Genomic differences revealed in a previous study showed that PR - Flavobacteriia have significantly larger genomes than PR + Flavobacteriia, although the ecophysiological reasons for this phenomenon remains enigmatic [11].

In this study, we sequenced 21 marine Flavobacteriia genomes and conducted comparative genomic analysis of 41 $\mathrm{PR}-$ and $35 \mathrm{PR}+$ marine Flavobacteriia. From a methodological perspective, our analysis was performed to fulfil two prerequisite conditions for successfully discovering systematic differences between different types of genomes [14, 15]. First, to attenuate strain-specific signals and achieve sufficient statistical power, a sufficiently large number of genomes that were not strongly biased within a single type are required. Second, genomes that belong to each different type need to be phylogenetically dispersed. Otherwise, genomic differences due to phylogenetic constraints (i.e., an effect that phylogenetically closely related species tend to have similar genomes just because they share a common ancestor) as opposed to ecophysiological adaptations, will bias the analysis.

\section{Materials and methods}

\section{Sample preparation and genome sequencing}

Supplementary Table S1 shows the summary of 21 marine Flavobacteriia strains whose genomes were sequenced in this study. Seven Polaribacter (P. butkevichii KCTC $12100^{\mathrm{T}}, P$. gangjinensis KCTC $22729^{\mathrm{T}}, P$. glomeratus ATCC $43844^{\mathrm{T}}$, P. sejongensis KCTC $23670^{\mathrm{T}}$, P. reichenbachii KCTC $23969^{\mathrm{T}}$, P. porphyrae NBRC $108759^{\mathrm{T}}$, and $P$. filamentus ATCC $700397^{\mathrm{T}}$ ) and six Nonlabens (N. agnitus JCM $17109^{\mathrm{T}}, N$. arenilitoris KCTC $32109^{\mathrm{T}}, N$. sediminis NBRC $100970^{\mathrm{T}}, N$. spongiae JCM $13191^{\mathrm{T}}, N$. tegetincola JCM $12886^{\mathrm{T}}$, and $N$. xylanidelens DSM $16809^{\mathrm{T}}$ ) type strains were provided by the NITE Biological Resource Center (NBRC), Japan Collection of Microorganisms (JCM), American Type Culture Collection (ATCC), Korean Collection for Type Cultures (KCTC), and Deutsche Sammlung von Mikroorganismen und Zellkulturen $\mathrm{GmbH}$ (DSMZ). The other eight strains were isolated from environmental samples in 2009 [3]: four strains from surface seawater at Western North Pacific Station $S$ $\left(30^{\circ} 40^{\prime} \mathrm{N}, 138^{\circ} 00^{\prime} \mathrm{E}\right)$ during cruise KT-09-11 of the R/V 'Tansei Maru' (Atmosphere and Ocean Research Institute, The University of Tokyo and Japan Agency for MarineEarth Science and Technology (JAMSTEC)) (Aureicoccus marinus SG-18 ${ }^{\mathrm{T}}$, Tenacibaculum sp. SG-28, Tenacibaculum sp. SZ-18, and Gilvibacter sp. SZ-19), one strain from surface seawater at Western North Pacific Station S1 $\left(30^{\circ} 11^{\prime} \mathrm{N}, 145^{\circ} 05^{\prime} \mathrm{E}\right)$ during cruise MR10-01 of the R/V 'Mirai' (JAMSTEC) (Aureitalea marina NBRC 107741 ${ }^{\mathrm{T}}$ ), two strains from sea ice in Saroma-ko Lagoon $\left(44^{\circ} 07^{\prime} \mathrm{N}\right.$, $143^{\circ} 58^{\prime} \mathrm{E}$ ) (Polaribacter spp. SA4-10 and SA4-12), and one strain from surface seawater at Sagami Bay Station $\mathrm{P}$ $\left(35^{\circ} 00^{\prime} \mathrm{N}, 139^{\circ} 20^{\prime} \mathrm{E}\right.$ ) during cruise KT-09-11 (Winogradskyella sp. PC-19). All strains were cultivated using half strength ZoBell's 2216E medium.

Genomic DNA samples were extracted by the standard phenol-chloroform method [16]. Genomes of two strains were sequenced using a 454 FLX + System (Roche) and an Ion PGM System (Thermo Fisher Scientific) and assembled using the Newbler assembler v2.7 software (Roche). Genomes of 11 strains were sequenced using a 454 FLX+ System and a MiSeq (Illumina) platform and assembled using the Newbler assembler v2.7 software. Genomes of the other eight strains were sequenced using a PacBio RS II (Pacific Biosciences) instrument and assembled using Sprai v0.9.5.1.3 (http://zombie.cb.k.u-tokyo.ac.jp/sprai/) and subsequent manual curation. All sequencing was performed by following manufacturers' protocols, and all assembling steps were performed using default parameters.

\section{Data set preparation and assessment of genome completeness}

We downloaded 55 genomes of marine Flavobacteriia from the NCBI RefSeq database [17] (Supplementary Table S2). During the quality check of the sequenced genomes, we found that several scaffolds of $P$. sejongensis KCTC $23670^{\mathrm{T}}$ and $P$. reichenbachii KCTC $23969^{\mathrm{T}}$ genomes were likely to be contaminated. We randomly selected 10 protein-coding sequences (CDSs) from the six scaffolds that coded CDSs and identified their origins by sequence similarity searches against the UniProt database [18] (downloaded in April 2017, results in Supplementary Table S3). The origin of 
each scaffold was consistently estimated at the genus level, and only the largest scaffold from each genome was concluded to be from the Polaribacter strains. We assessed the completeness of these two genomes after removing the other scaffolds using Benchmarking Universal Single-Copy Orthologs (BUSCO) version 3.0.0 [19] and a 443 orthologue data set that is conserved in the class Bacteroidetes, and obtained high scores $(97.7 \%$ for $P$. sejongensis KCTC $23670^{\mathrm{T}}$ and $98.4 \%$ for $P$. reichenbachii KCTC $23969^{\mathrm{T}}$ ).

The completeness of all 76 genomes was also assessed using BUSCO on the Bacteroidetes orthologue data set. The scores averaged $98.0 \%$, and the completeness of five genomes was $<95.0 \%$. The lowest BUSCO score was that of Salinibacter ruber DSM $13855^{\mathrm{T}}$, which had acquired many genes from hyperhalophilic archaea [20]. Excluding the five genomes with $<95.0 \%$ completion did not affect the conclusions of this study.

\section{Functional annotation of genes}

All 76 genomes were annotated through the following procedure. Ribosomal and transfer RNA genes were annotated using RNAmmer v1.2 [21] and tRNAscan-SE v1.3.1 [22], respectively, with their default settings. Subsequently, we masked the rRNA and tRNA gene sequences with "N" and predicted CDSs using Prodigal v2.50 [23] at default settings.

The functional annotation of the 258,135 CDSs was performed by eggNOG-mapper [24] and the bactNOG data set in the eggNOG database version 4.5 [25], by adopting the DIAMOND option for mapping and by setting the taxonomic scope to Bacteroidetes. This approach resulted in functional annotation of 184,623 (71.5\%) of the CDSs to 14,361 eggNOG orthologue groups, excluding functionunknown orthologue groups (i.e., groups whose annotations contained any of the terms "NA", "unknown", or "DUF").

Amino-acid sequences of CDSs that were annotated to the rhodopsin orthologue group (ENOG05CSB) were aligned using MAFFT version 7.212 [26] with the linsi algorithm and default parameters. The alignments were curated using trimAl version 1.2 [27] with the option "-gt 1". The best substitution model of each alignment was selected using prottest3 [28]. The maximum-likelihood method was performed using RAxML version 7.2.8 [29] and 1000 bootstrap replicates. The other settings were set to their default values. Phylogenetic classification of rhodopsins as $\mathrm{PR}, \mathrm{Na}^{+}$-pumping rhodopsin $(\mathrm{NaR})$, and $\mathrm{Cl}$ - -pumping rhodopsin (CIR) genes was conducted as described in our previous study [30].

\section{Reconstruction of the genomic phylogenetic tree}

As outgroups, genomes of two strains of the class Bacteroidetes (Cytophaga hutchinsonii ATCC $33406^{\mathrm{T}}$ and
Salinibacter ruber DSM $13855^{\mathrm{T}}$ ) were additionally downloaded from the NCBI RefSeq database. The prediction and annotation of their CDSs were conducted in the same manner as the other genomes. We selected 155 ENOG orthologue groups so that each genome contained exactly one CDS that was annotated to each of those orthologue groups. Their amino-acid sequences were aligned using MAFFT and curated using trimAl as described above. The best substitution model of each alignment was selected by using prottest 3 . The alignments of 155 eggNOG orthologue groups were concatenated and subjected to phylogenetic tree reconstruction using RAxML with the best substitution model for each protein column and 1000 bootstrap replicates. The other settings were set to their default values.

\section{Genome size and gene content analysis}

The difference in the sizes of the PR - and $\mathrm{PR}+$ genomes was statistically evaluated by applying Student's $t$-test to the total scaffold sizes of the two groups. Orthologue group distributions that were biased in $\mathrm{PR}-$ or $\mathrm{PR}+$ genomes were identified by applying the Brunner-Munzel test [31] to the numbers of CDSs in each of the 14,361 eggNOG orthologue groups. To correct for multiple testing, Storey's approach [32] was used with a cut-off false discovery rate of 0.05 .

For the orthologue group distribution analyses across different phyla, the eggNOG 4.5 database [25] and Microbial Genome Database for Comparative Analysis (MBGD) database, updated on 2015 April, was used.

\section{Experimental analysis of DUF2237}

The DUF2237 gene of Synechocystis sp. PCC 6803-P (i.e., slr1628) [33] was inactivated by replacing it with a chloramphenicol resistance cassette. A DNA sequence that contained the region that is 500-bp upstream of the DUF2237 gene, a chloramphenicol resistance cassette, and 500-bp downstream of DUF2237 was artificially synthesized and inserted into a pEX-A2 vector (Eurofins Genomics). Knockout strains were generated by transforming this plasmid into PCC 6803-P cells, growing these cells at $30{ }^{\circ} \mathrm{C}$ under continuous white light with an intensity of $50 \mu \mathrm{mol} \mathrm{m}$ ${ }^{-2} \mathrm{~s}^{-1}$, and selecting colonies on plates with BG-11 medium [34] that contains $20 \mu \mathrm{g} \mathrm{m} 1^{-1}$ chloramphenicol. Because PCC 6803-P cells contain multiple genomes in each cell, the segregation between the wild-type and DUF2237-knockout genomes was examined by PCR with DUF2237-upstream (5'-AATCTCTGCTAGGTTTGG-3') and DUF2237downstream (5'-AACTCTGGTAGCTGTTCC-3') primers after 3 days of growth on the BG-11 plates.

For the phototaxis assay, wild-type and DUF2237knockout cells were collected in the exponential phase, 
suspended in BG-11 liquid medium at an optical density of 0.1 , and spotted onto $1.5 \%$ agarose BG-11 plates four times per strain. The spotted plates were incubated under unidirectional white light with an intensity of $22 \mu \mathrm{mol} \mathrm{m}{ }^{-2} \mathrm{~s}^{-1}$ at $30^{\circ} \mathrm{C}$ for 7 days, and the distances of colony movements were measured.

\section{Analysis of RNR gene classes}

To identify the classes of RNR genes, all CDSs that were annotated with the ENOG05BZH were fed into domainlevel annotation using the NCBI conserved domain search [35]. For phylogenetic analysis of RNR genes, RNR genes of Lactobacillus leichmannii (GenBank: AAA03078) and Escherichia coli H736 (GenBank: EGI11882) were downloaded from GenBank to serve as representatives of class II and class I genes, respectively. CDSs were aligned, and the alignments were curated by the same methods described above. The best-fit substitution model was selected using prottest 3 at its default settings. The maximum-likelihood method was performed using RAxML and 1000 bootstrap replicates. The other settings were set to their default values.

\section{Analysis of Tara Oceans data set}

The Tara Oceans data set, containing gene abundance FPKM value, oxygen concentration, and sampling depth data, was downloaded from http://ocean-microbiome.embl. de/companion.html [36]. Correlation analysis was conducted using the "psych" package in R (https://pbil.univlyon1.fr/CRAN/web/packages/psych/). Curve fitting was done using locally weighted scatterplot smoothing with its default options.

\section{Results}

\section{Marine Flavobacteriia genome sequencing and data set preparation}

To obtain a large, unbiased, and polyphyletic (phylogenetically dispersed) genomic data set, genomes of 21 marine Flavobacteriia strains were sequenced. These strains contained seven Polaribacter type strains, six Nonlabens type strains, and eight strains that were isolated from Saroma-ko Lagoon (Hokkaido, Japan), Sagami Bay (Kanagawa, Japan), and the western North Pacific Ocean (Supplementary Table S1). We subsequently downloaded 55 genomes of marine Flavobacteriia from the NCBI RefSeq database [17] and constructed a genomic data set of 76 marine Flavobacteriia strains, 41 and 35 of which were $\mathrm{PR}-$ and $\mathrm{PR}+$ strains, respectively (Supplementary Table S2; their sampling sites are shown in Supplementary Fig. S1). All genomes were subjected to in-house annotation of their ribosomal RNAs, transfer RNAs, and CDSs. To evaluate the quality of the 76 genomes, their completeness was estimated using BUSCO (version 3.0.0) software [19]. All but five genomes were $>95 \%$ complete by this metric. Excluding those five genomes from the analyses did not affect the conclusions of this study.

\section{Functional annotation and confirmation of polyphyletic PR distribution}

The CDSs were functionally annotated using eggNOGmapper [24] and the bactNOG data set in the eggNOG database [25]. Among the 258,135 CDSs in total, 71.5\% were assigned to any eggNOG orthologue group by ignoring function-unknown groups. We further classified the CDSs that were assigned to the rhodopsin orthologue group (ENOG05CSB) as PR, NaR, and ClR genes by phylogenetic analysis (Supplementary Fig. S2). Whereas all NaRpossessing strains had additional PR genes, two CIRpossessing strains (Nonlabens spongiae $\mathrm{JCM} 13191^{\mathrm{T}}$ and Psychroserpens sp. Hel_I_66) were revealed to lack PR genes. We treated these two CIR-possessing strains as PR+ strains in the following analyses because the inward $\mathrm{Cl}$ -pumping activity of $\mathrm{ClR}$ also generates membrane potential; however, the conclusions were not affected even if they were treated as PR- strains.

We then reconstructed a genomic phylogenetic tree of the 76 marine Flavobacteriia strains by applying the maximum-likelihood method to the concatenated protein sequence data set of 155 conserved CDSs that were present in each strain in exactly one copy. To root the tree, genomes of two strains of the phylum Bacteroidetes were added to the data set as outgroups. We confirmed that the PR - and $\mathrm{PR}+$ strains had polyphyletic distributions on the reconstructed phylogenetic tree, fulfilling the second condition for a comparative genomic study (Fig. 1).

\section{Detection of genes significantly biased in either PR- or $\mathrm{PR}+$ genomes}

We first compared the genome sizes of PR - and PR+ marine Flavobacteriia strains. As consistent with previous findings [11], the PR - genomes were significantly larger than the PR + genomes ( $p$-value $=4.7 \mathrm{E}-3$, Figs. 1, 2a). To further investigate the ecophysiological background that caused this difference in genome size, we compared their CDS numbers in each eggNOG functional category [25] (Fig. 2b). We discovered that except for several categories that are generally rare in bacteria, the numbers of CDSs were consistently larger in the PR - genomes than in the $\mathrm{PR}+$ genomes, regardless of their functional categories. This result suggests that the observed genome size 


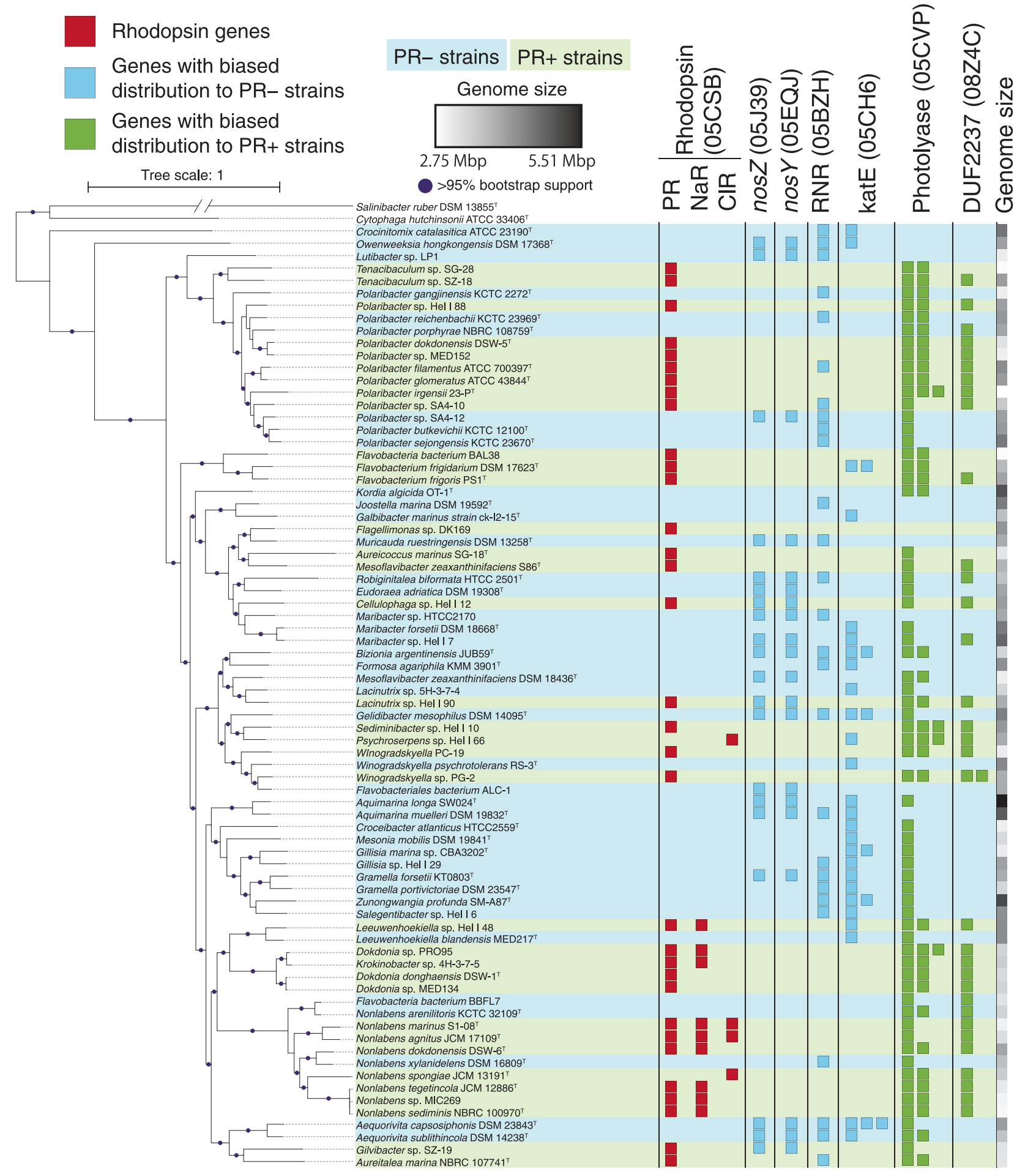

Fig. 1 Phylogenetic tree and distributions of genes biased in PR - or $\mathrm{PR}+$ genomes. (Left) A maximum-likelihood genomic tree based on 155 CDSs that were conserved across the 76 marine Flavobacteriia genomes. The closed circles indicate branches with $95 \%$ bootstrapping support. Yellow and purple background colours indicate PR - and PR + strains, respectively. The tree was visualized using iTol v 3.3.2 [71]. (Right) The number of genes coded by each genome is represented by the numbers of closed squares. Red: Rhodopsin genes. Light blue:
eggNOG orthologue groups that showed distributions that were particularly biased in $\mathrm{PR}-$ genomes. Light green: those particularly biased in PR+ genomes. For RNR (05BZH) and photolyase (05CVP) orthologs, one gene in each strain is not shown because all strains except for Lutibacter sp. LP1 possess at least one copy of those orthologs. Note that distributions of genes for the synthesis and transport of APEs are shown in Fig. 5. The genome size of each strain was indicated in grey scale 


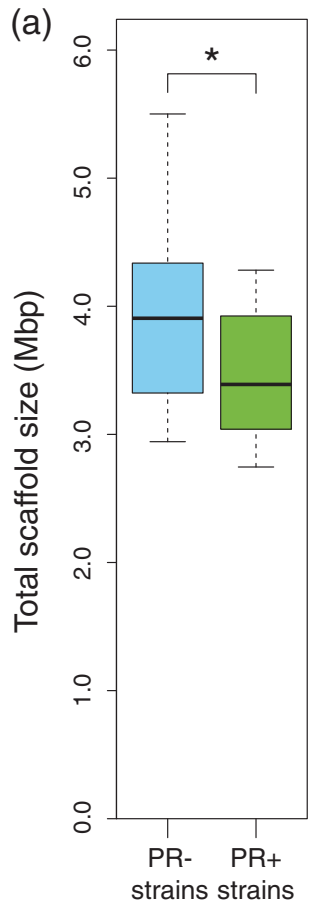

Fig. 2 Genome sizes and quantities of CDSs in PR - and PR+ marine Flavobacteriia. a Total scaffold sizes of PR - (blue) and PR+ (green) genomes. The bottom, central line, and top of the box plots represent the first, second, and third interquartile ranges (IQR), respectively.

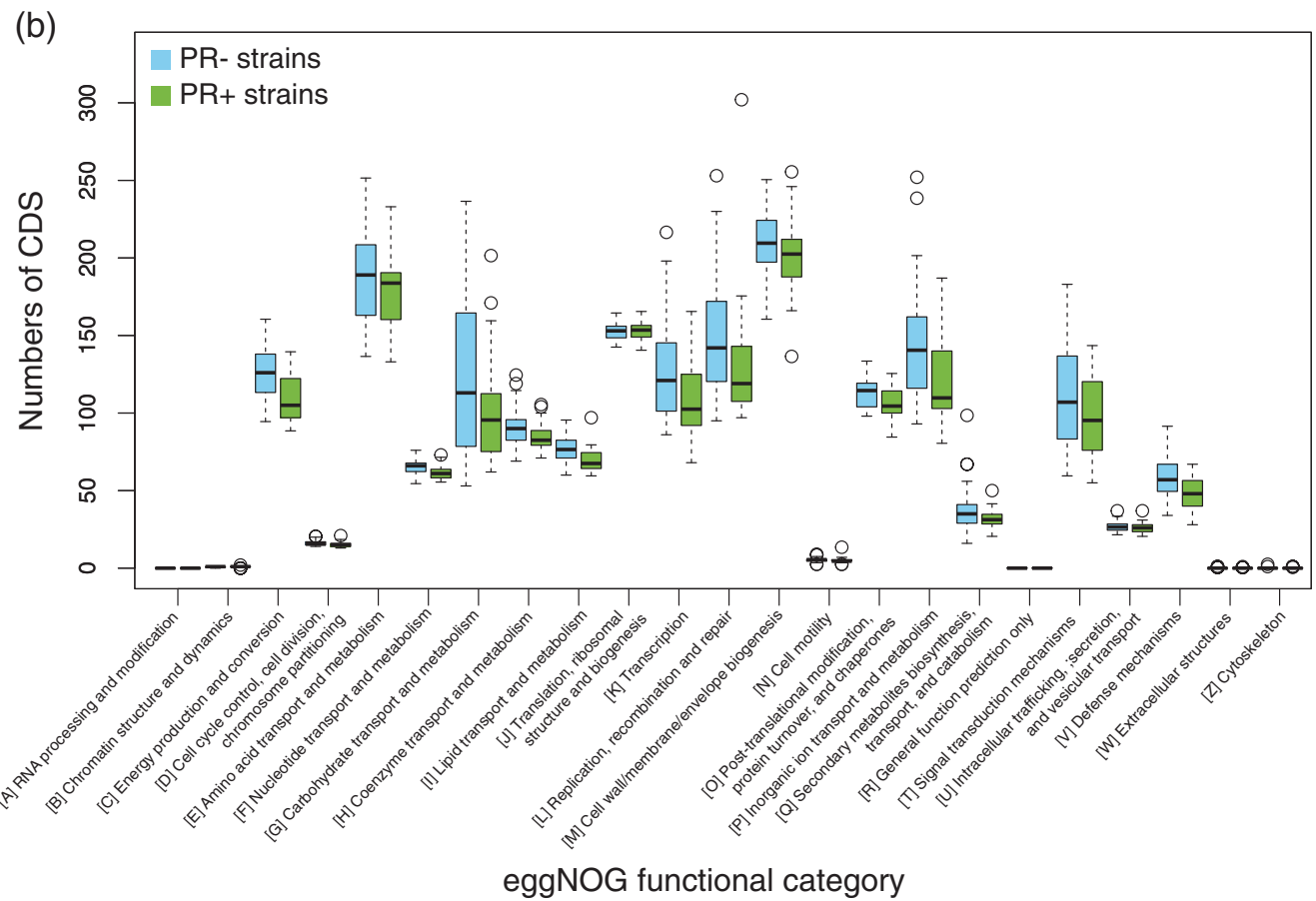

Whiskers represent the lowest and highest values within $1.5 \times \mathrm{IQR}$ from the first and third quartiles, respectively. b Quantities of CDSs in each eggNOG functional category in the box plot drawn in the same manner. Circles represent outliers beyond the whiskers

pattern of those genes, we assume that the fixation of inorganic carbonic acid by anaplerotic carbon fixation would be a common feature among marine Flavobacteriia.

\section{Experimental analysis of a function-unknown gene strongly biased in PR+ genomes}

The orthologue group that showed the second most biased distribution contained the DUF2237 genes (q-value $=3.9 \mathrm{E}$ 10), which were function-unknown genes that were enriched in the PR+ genomes (Fig. 1 and Supplementary Table S5). Using the MBGD [37], we found that DUF2237 genes (MBGD ID 4444) are broadly distributed across 11 phyla, and many Cyanobacteria, phototrophic bacteria, and rhodopsin-containing Euryarchaeota have this gene. The sequence of the DUF2237 gene is highly conserved across different phyla (Fig. 3a). MBGD analysis showed that DUF2237 is possessed by $72 \%$ and $66 \%$ of prokaryotes that have photosystem II (pufM/psbA/pufL, MBGD ID 2841) and rhodopsin genes (MBGD ID 22185 and 4672), respectively, whereas only $17 \%$ of all prokaryotes have DUF2237 (Fig. 3b). This bias was not just because Cyanobacteria tend to have DUF2237 (i.e., phylogenetic constraint); we confirmed that excluding Cyanobacteria did not diminish the observed bias (Fig. 3b). These observations strongly suggested that DUF2237 has a widely conserved function that is related to general phototrophy. 
(a)

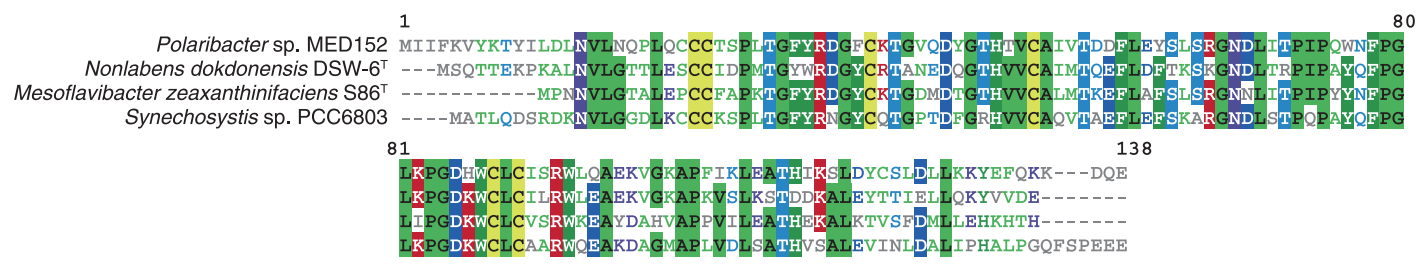

(b)

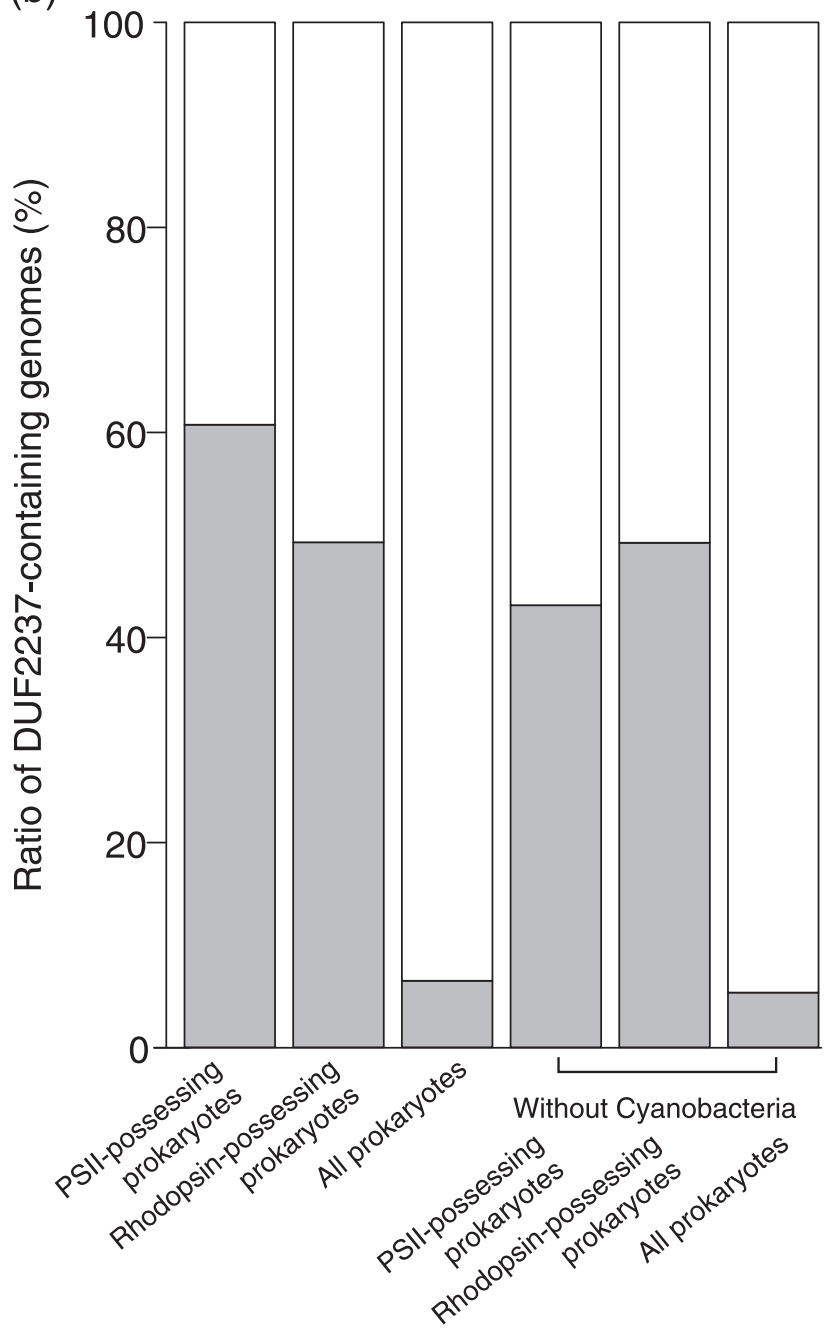

(c)

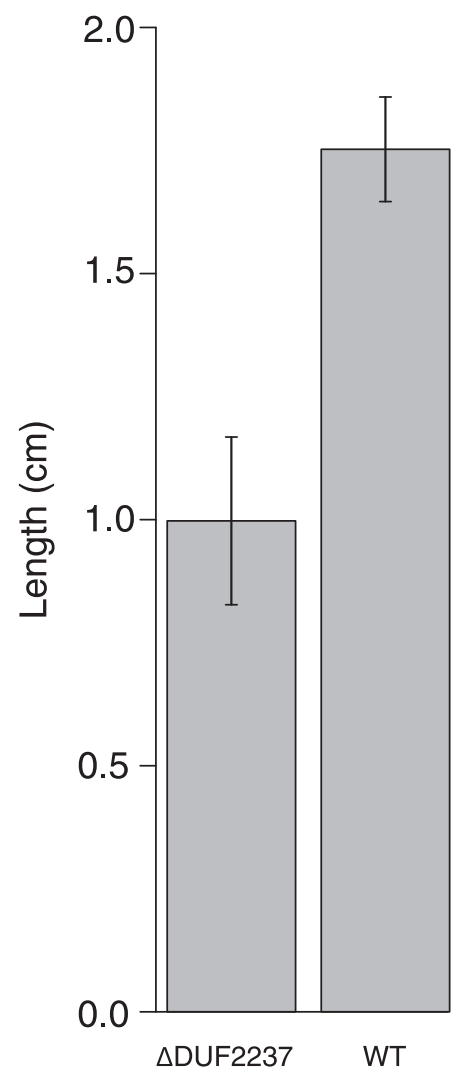

Fig. 3 Analysis of the DUF2237 gene. a Multiple alignment of DUF2237 amino-acid sequences of Flavobacteriia strains and Synechocystis sp. PCC6803. Amino acids with background colours indicate residues with $>50 \%$ consensus. The multiple alignment was conducted by using MAFFT with the linsi algorithm and its default options and was visualized by using MView [72]. b Biased distribution of DUF2237 genes to phototrophs. The bar chart represents the ratios of DUF2237-possessing strains in the PSII-possessing prokaryotes $(n=$ $51)$, rhodopsin-possessing prokaryotes $(n=52)$, all prokaryotes $(n=$

To experimentally confirm the functional importance of DUF2237, we knocked its gene out of Synechocystis sp. PCC6803-P [38]. We selected this cyanobacterial strain because it has a DUF2237 gene and methods to manipulate
547), PSII-possessing non-cyanobacterial prokaryotes $(n=20)$, rhodopsin-possessing non-cyanobacterial prokaryotes $(n=44)$, and all non-cyanobacterial prokaryotes $(n=515)$. The data were obtained from the MBGD database. c Phototaxis assay of the DUF2237 gene knockout strain of Synechocystis sp. PCC6803-P. The bar graph shows the distances of colony movements from the spotted points under unidirectional light. Four replicated experiments were conducted for each strain. Statistical significance was examined by Student's $t$-test ( $p$ value $=2.9 \mathrm{E}-4)$

its genome are well established. The DUF2237-knockout strain did not show any apparent difference in proliferation speed and other phenotypes under standard laboratory culture conditions; however, in phototaxis assays, the 
DUF2237-knockout strain showed significantly less movement than the wild-type strain, which exhibits positive phototaxis under unidirectional white light ( $p$-value $=$ 2.9E-4, Fig. 3c). This result is consistent with the strong correlation between the presence of DUF2237 and phototrophy because phototaxis should be beneficial to organisms that utilize light. Although cyanobacterial phototaxis is a phenotype in which many proteins are involved (e.g., light sensing, signal transduction, transcriptional regulation, and pilus formation proteins) [38-40] and further analyses are required to clarify the molecular basis of the DUF2237 function, this result proves that our comparative genomics approach is powerful enough to find genes that reflect microbial ecophysiology.

\section{Proximity analysis of genes biased in PR- or PR+ genomes}

We conducted gene proximity analysis of the 129 orthologue groups that showed biased distributions in PR- or PR+ strains because genes that are near each other in genomes likely have related functions [41]. A gene proximity network was constructed by connecting any orthologue group pair that are located within $20 \mathrm{~kb}$ of each other in at least 10 genomes in our data set (Fig. 4). A typical example of such proximal relation was seen between the rhodopsin and blh genes, which are often adjacently coded for concerted expression [42]. We note that the two ClR-possessing PRstrains code the blh genes next to their ClR genes.

Three large clusters were formed in the gene proximity network. Among them, two clusters were composed of genes that were enriched in the PR-genomes: the first was composed of genes for anaerobic nitrous oxide metabolism, and the second was composed of genes for synthesis and transport of aryl polyenes (APEs). The third cluster was composed of photolyase and photolyase-related genes, which were enriched in the $\mathrm{PR}+$ genomes. These three large clusters were assumed to especially reflect lifestyles to which PR - and PR+ Flavobacteriia species have adapted.

\section{Signs of adaptation of PR- Flavobacteriia to anaerobic conditions}

Despite a predominance of function-unknown genes in the 129 orthologue groups that showed biased distributions, we discovered one interesting trend therein: the genes enriched in the PR - genomes showed several signs of adaptation to microaerobic or anaerobic conditions although Flavobacteriia species are usually considered to be (strictly) aerobic [43].

The PR - genomes coded significantly more nitrous oxide reductase (nos $Z$, ENOG05EQJ) and nitrous oxide metabolism (nosY, ENOG05J39) genes than the $\mathrm{PR}+$ genomes (Fig. 1, q-value $=3.7 \mathrm{E}-2$ and 3.7E-2, respectively). These genes, which were members of the first cluster that was formed in the gene proximity network (Fig. 4), function in bacterial anaerobic $\mathrm{N}_{2} \mathrm{O}$ respiration [44, 45], which uses nitrous oxide as a terminal electron acceptor at reduced oxygen concentrations [46]. Second, the PRgenomes had more class II ribonucleotide reductase (RNR) genes $($ ENOG05BZH) (Fig. 1, q-value =9.9E-5). RNR proteins catalyze the synthesis of deoxyribonucleotides from ribonucleotides and are grouped into three classes according to their subunit types [47]. NCBI conserved domain searches [35] and a phylogenetic analysis (Supplementary Fig. S4) showed significant enrichment of the class II RNR genes in the PR - genomes (PR - : 23/41, PR $+: 2 / 35$ ) occurred. Class II RNRs do not depend on oxygen for their catalytic function, whereas class I RNRs function under aerobic conditions [47]. A catalase gene, katE (ENOG05CH6), was also enriched in the PR - genomes (Fig. 1, q-value $=4.9 \mathrm{E}-3$ ). This gene was reported to modulate reactive oxygen stress when cells that usually live in anaerobic conditions are exposed to oxygen. Expression of katE increases under anaerobic conditions in E. coli [48], and the kat $E$ protein is the only $\mathrm{H}_{2} \mathrm{O}_{2}$-removing enzyme that is present in an obligate anaerobic Bacteroidetes, Bacteroides thetaiotaomicron [49, 50]. In addition, the PRgenomes almost always had $c b b_{3}$-type cytochrome oxidase genes (ENOG05EUH), whereas the PR + genomes did not (Supplementary Fig. S5, q-value $=1.6 \mathrm{E}-2$ ). The $c b b_{3}$-type cytochrome oxidases have a very high affinity for $\mathrm{O}_{2}$ so that their organisms can respire under microaerobic conditions [51], and they should enable Flavobacteriia to survive in transiently low- $\mathrm{O}_{2}$ microniches [52].

\section{Enrichment of ultraviolet (UV)-screening pigment synthesis genes in PR- genomes}

The second cluster in the gene proximity network contained 16 genes for the synthesis and transport of APEs and was enriched in the PR- genomes (Fig. 4). Most notably, almost all genes in this cluster were not only significantly but also exclusively found in the PR - genomes (Fig. 5a). The genes in this cluster corresponded well to those previously reported in an APE-producing gene cluster in the Flavobacterium johnsoniae ATCC $17061^{\mathrm{T}}$ genome [53] (Fig. 5b). These data strongly suggested that production of APEs is a unique feature of $\mathrm{PR}$ - marine Flavobacteriia.

APEs (Fig. 5c) protect bacterial cells from UV and visible light by localizing to outer membranes [54, 55]. This localization to outer membranes contrasts with the localization of carotenoids to inner membranes [56] but resembles that of scytonemin, a cyanobacterial UV-screening extracellular phenolic pigment [57]. When proteins that synthesize the dialkylresorcinol (DAR) moiety are present 


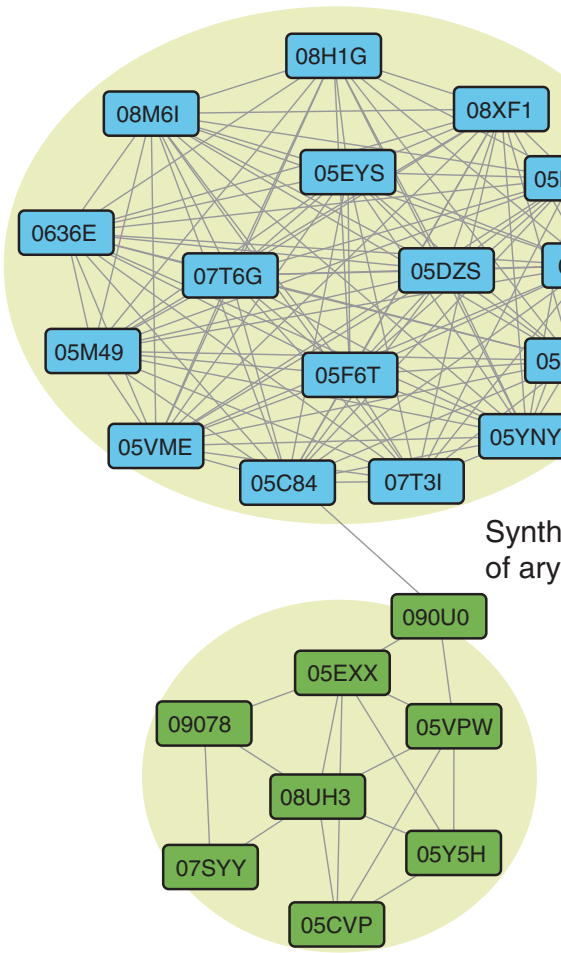

Photolyase and related genes

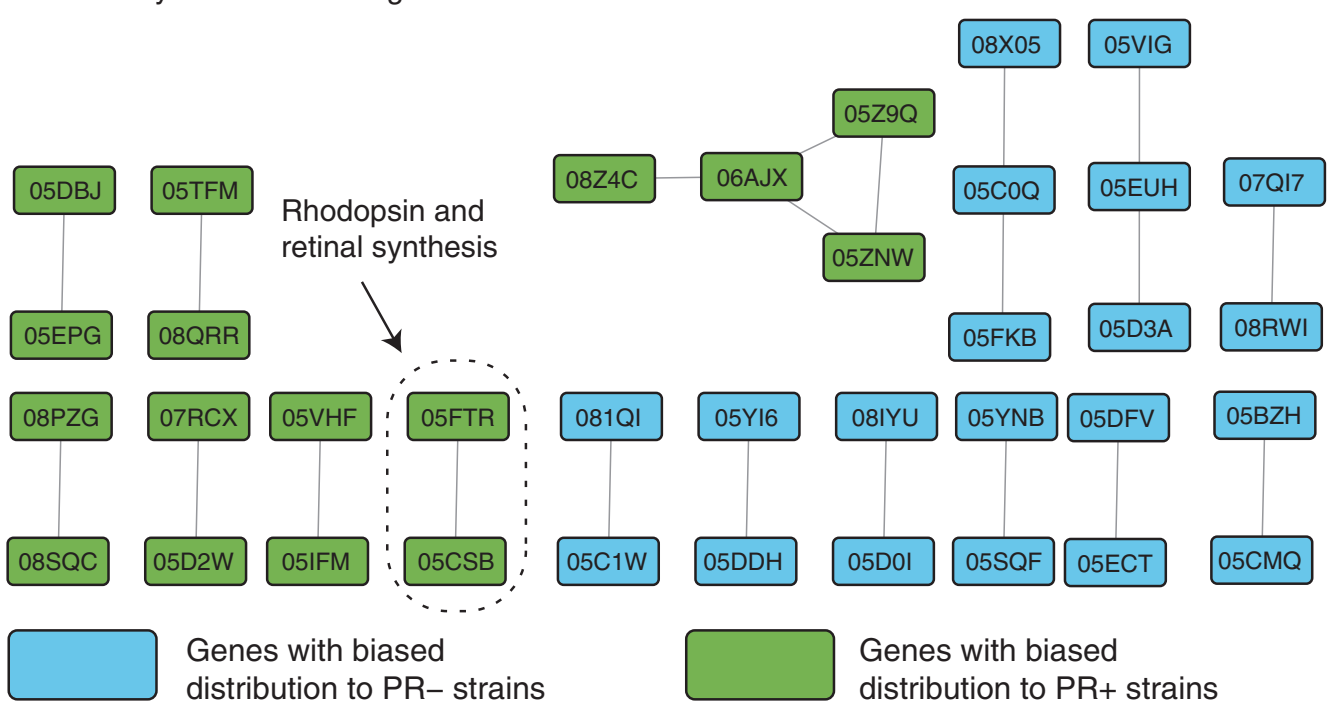

Fig. 4 Gene proximity network of orthologue groups that showed biased distributions. Boxes in light blue and light green represent eggNOG orthologue groups that are biased in the PR - and PR+ genomes, respectively. Note that 49 of the 129 orthologue groups

(e.g., in $F$. johnsoniae cells), APEs are esterified with the DAR moiety and converted to flexirubin-type pigments (FTPs) (Fig. 5d). FTPs are well-studied yellow-to-orange pigments specific to Bacteroidetes and have been used as a chemosystematic marker for taxonomic studies because of its polyphyletic distribution [58, 59]. FTPs also absorb UV and visible light $[59,60]$ and localize to outer membranes [56], and can be detected by a flexirubin test [58]. Thus, we conducted a flexirubin test on Aequorivita capsosiphonis

showed no proximal relations and are absent from this figure. Three large clusters are indicated by the light-yellow ellipses. The PR and blh genes are indicated by the ellipse with a dashed border. Gene annotations are available in Supplementary Tables S4 and S5

DSM $23843^{\mathrm{T}}$, whose genome codes APE synthesis genes and the darA (ENOG08K4P) and darB (ENOG05CXX) genes that are used to synthesize the DAR moiety (Fig. 5a). This test experimentally confirmed that this strain actually synthesizes FTPs (data not shown). Another strain that has these genes, Aquimarina muelleri DSM $19832^{\mathrm{T}}$, was also reported to respond positively to the flexirubin test [61].

Finally, the third cluster formed in the gene proximity network contained photolyase and photolyase-related genes 
(a)

\section{Rhodopsin genes}

Genes with biased significantly distribution to PR- strains Genes with no significantly biased distribution to PR+ or PR- strains

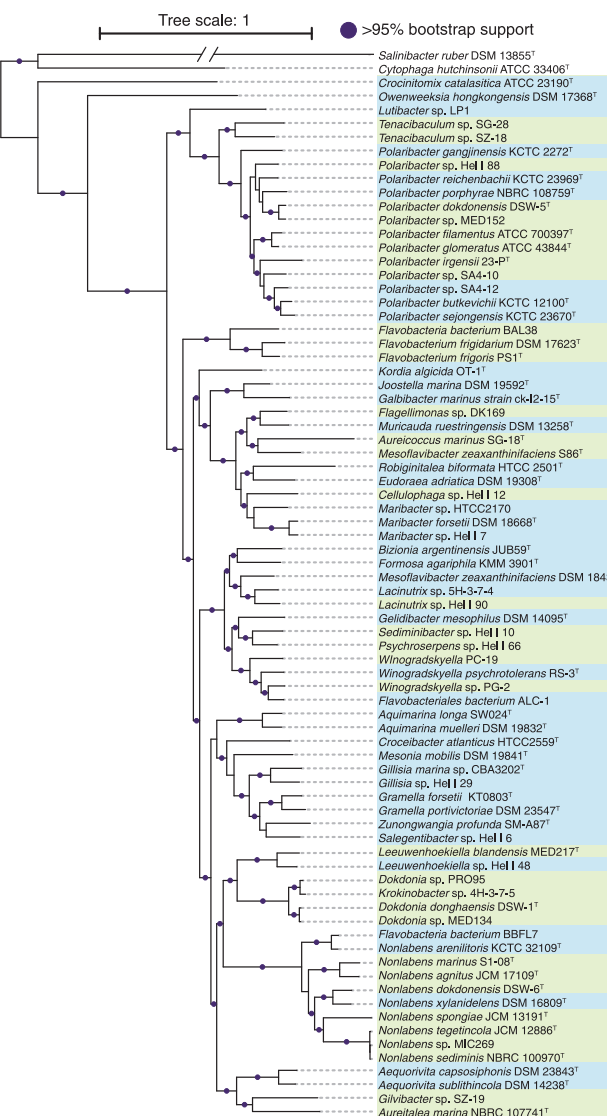

(b)

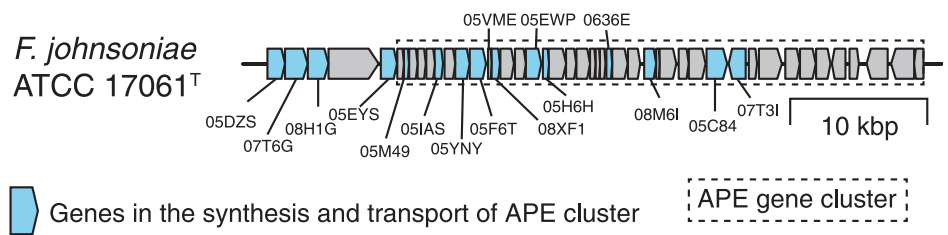

PR+ strains 유ㅇㅝㅔ
PR-strains $\frac{\text { ( }}{\bar{N}}$ Genes for DAR moiety synthesis Genes in the synthesis and transport of APE cluster

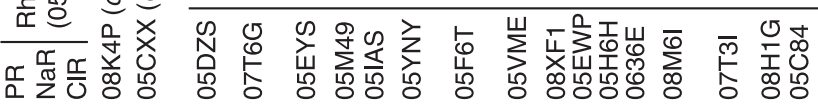

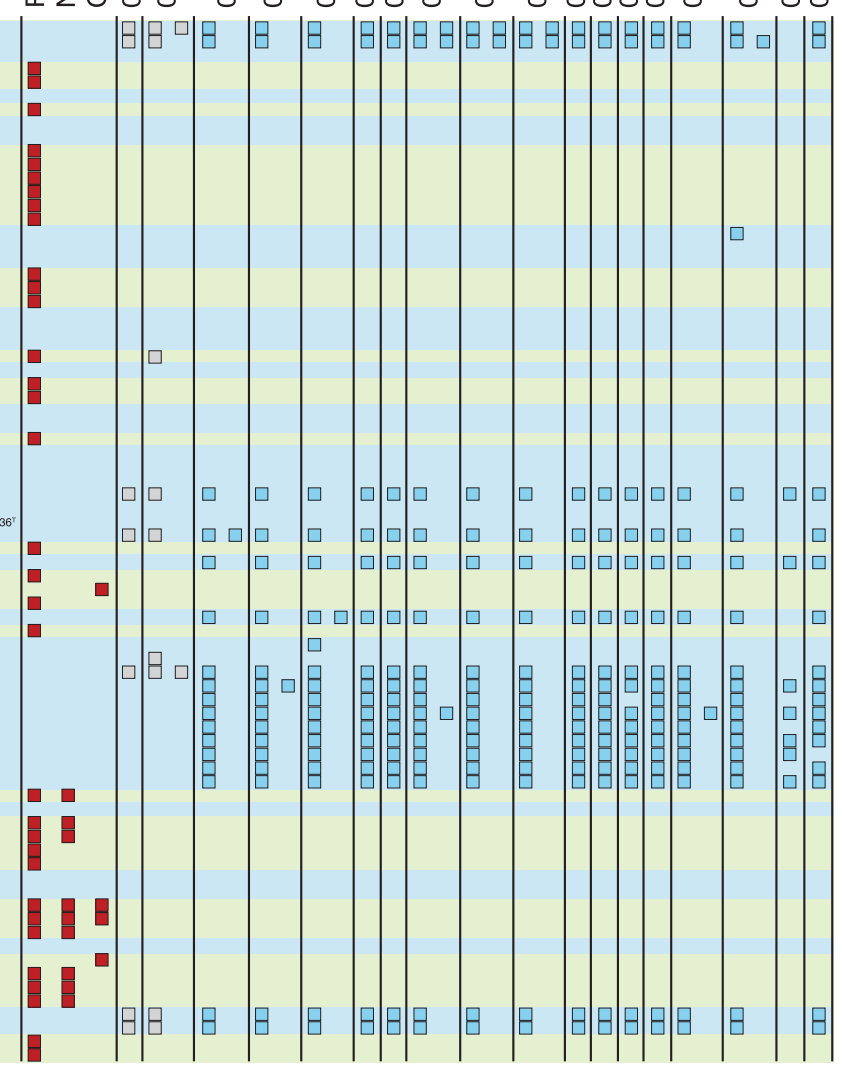

(c) APE (xanthomonadin)

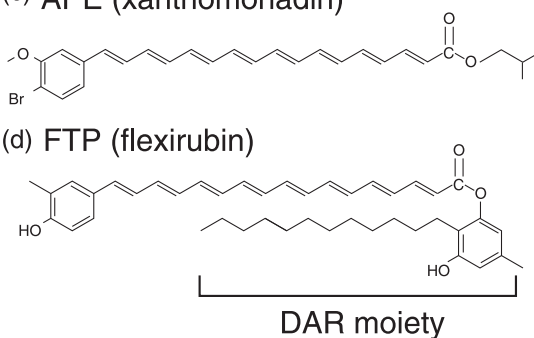

Fig. 5 Analysis of genes involved in the synthesis and transport of APEs. a Distributions of genes involved in synthesis and transport of APEs and DAR. The genomic phylogenetic tree is from Fig. 1. The closed circles indicate branches with $95 \%$ bootstrapping support. Yellow and purple background colours indicate PR - and PR + strains, respectively. Number of genes coded by each genome is illustrated by the number of closed squares. Red: rhodopsin genes. Grey: eggNOG orthologue groups involved in the synthesis of the DAR moiety (05CXX: darB and 08K4P: darA) and did not show biased distributions. Light blue: eggNOG orthologue groups in the cluster related to the synthesis and transport of APEs (05DZS: phenylacetate-CoA ligase, 07T6G: acyl-coenzyme A 6-aminopenicillanic acid acyltransferase, 05EYS: glycosyl transferase, family 2, 05M49: dehydratase, 05IAS: outer membrane lipoprotein carrier protein LolA, 05YNY: synthase, 05F6T: synthase, 05VME, acyl carrier protein,
08XF1: NA, 05EWP: synthase, 05H6H: thioesterase, 0636E: flexirubin-type pigment biosynthesis acyl carrier protein, 08M6I: lipid A biosynthesis acyltransferase, 07T31: 5'-nucleotidase, 08H1G: phospholipid glycerol acyltransferase, and 05C84: histidine ammonialyase) that showed distributions that were significantly biased in the PR- genomes. b Syntenic map of the APE gene cluster of $F$. johnsoniae ATCC $17061^{\mathrm{T}}$. Pentagons represent genes, and pentagon lengths are proportional to the gene lengths. Genes in the cluster related to the synthesis and transport of APEs in Fig. 3 are shown in light blue. The previously reported APE gene cluster of $F$. johnsoniae ATCC $17061^{\mathrm{T}}$ (between Fjoh_1080 and Fjoh_1115 genes) is represented by a rectangle with a dashed border. c Structure of a representative APE molecule (xanthomonadin). d Structure of a representative FTP molecule (flexirubin) 


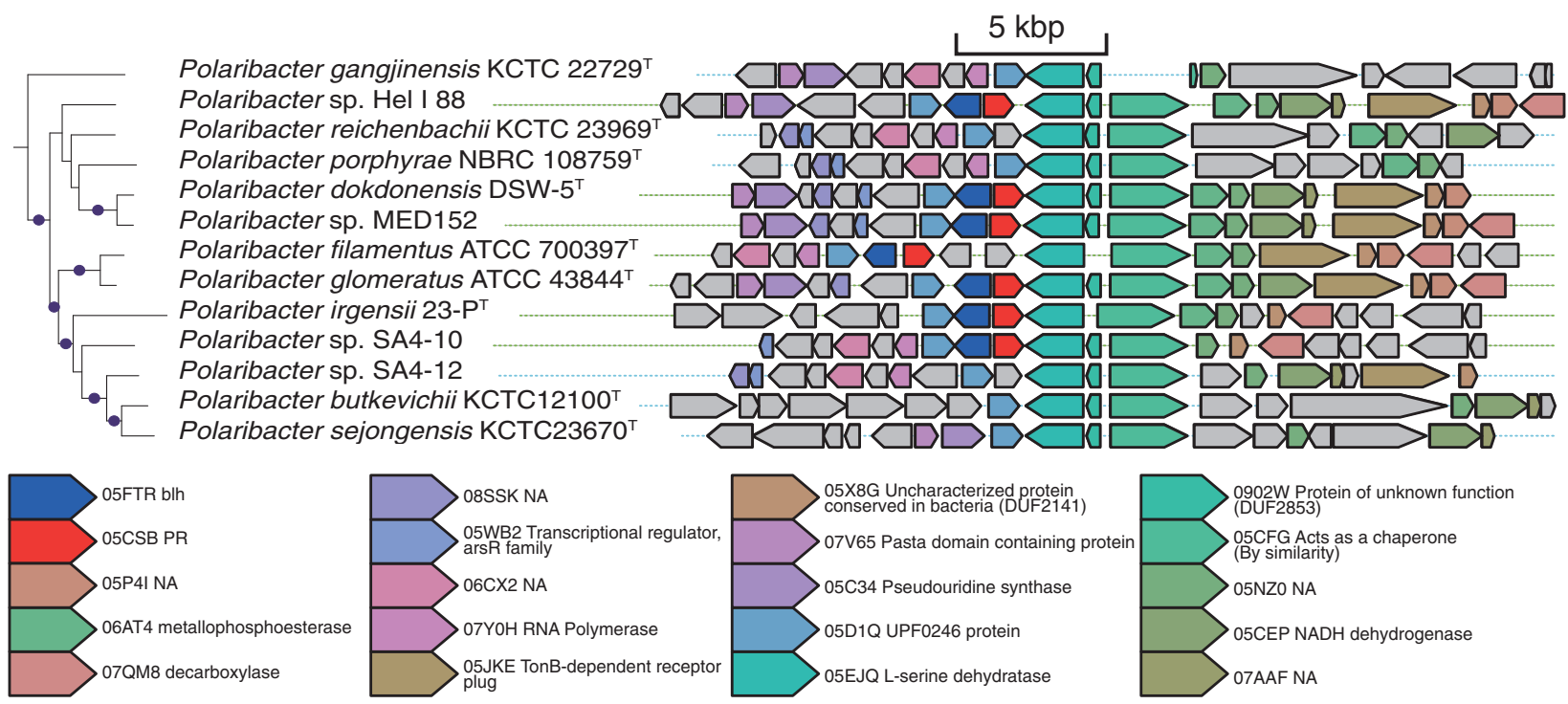

Fig. 6 Syntenic map of the region around PR genes in the Polaribacter genomes. A part of the genomic phylogenetic tree is from Fig. 1. The closed circles indicate branches with $95 \%$ bootstrapping support. Blue and green horizontal dotted lines indicate $\mathrm{PR}-$ and $\mathrm{PR}+$ strains, respectively. Pentagons represent genes, and pentagon lengths are proportional to the gene lengths. The conserved genes are shown in colours

and was enriched in the PR+ genomes (Fig. 4). Photolyase is an enzyme that uses visible light energy to repair DNA damage caused by UV light [62]. Specifically, the PR+ genomes coded significantly more genes of a photolyase paralogue (ENOG05CVP) than the PR- genomes did (Fig. 1, PR-: 1.9, PR+: 2.9 copies per genome on average).

\section{Discussion}

In this study, we conducted a comparative genomic analysis of $\mathrm{PR}-$ and $\mathrm{PR}+$ marine Flavobacteriia. The large and unbiased genomic data set enabled us to clarify their differences, which appear to be related to fundamentally different lifestyles and ecophysiological strategies. In addition, the polyphyletic distribution of PR genes (Fig. 1) and genomic traces indicated that PR genes have not only been gained but also lost during evolution (Fig. 6), suggesting that the conditions that have made each of the PR- and PR + lifestyles advantageous have not been stable during the course of evolution. The approach adopted in this study can be further applied to provide broader insights into microbial ecology in the future-the more genomes we have, the more powerful comparative genomic approaches become.

Although it might still be possible that the exclusive distribution pattern between the pigment synthesis and PR genes is due to unknown molecular mechanisms that prevent their co-existence, our results strongly suggest that PR - and PR+ marine Flavobacteriia adopt contrasting strategies to address UV damage: the former produces APEs or based on their annotation. Other genes are shown in grey. NA: Not Annotated. Because it is highly unlikely that the PR and blh genes were independently and repeatedly acquired next to the L-serine dehydratase gene by chance, the PR and blh genes are assumed to have been lost during evolution

FTPs to avoid UV damage, whereas the latter produces photolyase to efficiently repair themselves after UV damage (Fig. 7). We propose that PR+ Flavobacteriia accept both UV damage and cost of repairing UV-damaged DNA so that they can take advantage of light energy by using PR in their inner membranes. On the other hand, PR- Flavobacteriia avoid the UV damage by blocking the UV light and thus must abandon utilizing light energy. To confirm the generality of our finding across different taxonomic groups, we analyzed the distribution patterns of rhodopsin and APE synthesis genes in all prokaryotes. While both rhodopsin and APE synthesis genes are distributed across diverse phyla, we observed their completely exclusive distribution patterns, that is, no strain possesses both rhodopsin and APE genes (Table 1). In accord with the analogy in which PR functions as microbial "solar panels", we propose a "solar-panel or parasol" hypothesis, in which APEs and FTPs are regarded as cellular "parasols". In this framework, we can choose to either charge solar-powered devices or use parasols to avoid tanning but cannot do both simultaneously.

Notably, these two different strategies for the handling of UV damage may also explain the smaller genome size of $\mathrm{PR}+$ Flavobacteriia. First, UV damage itself would accelerate the net rate of genome size reduction in the PR+ strains via induced double-strand breaks and nonsense mutations [63]. Second, stronger selection pressure to minimize the DNA repair cost would also lead to the smaller genome size in PR+ Flavobacteriia. In contrast, $\mathrm{PR}$ - Flavobacteriia would receive less DNA damage and 


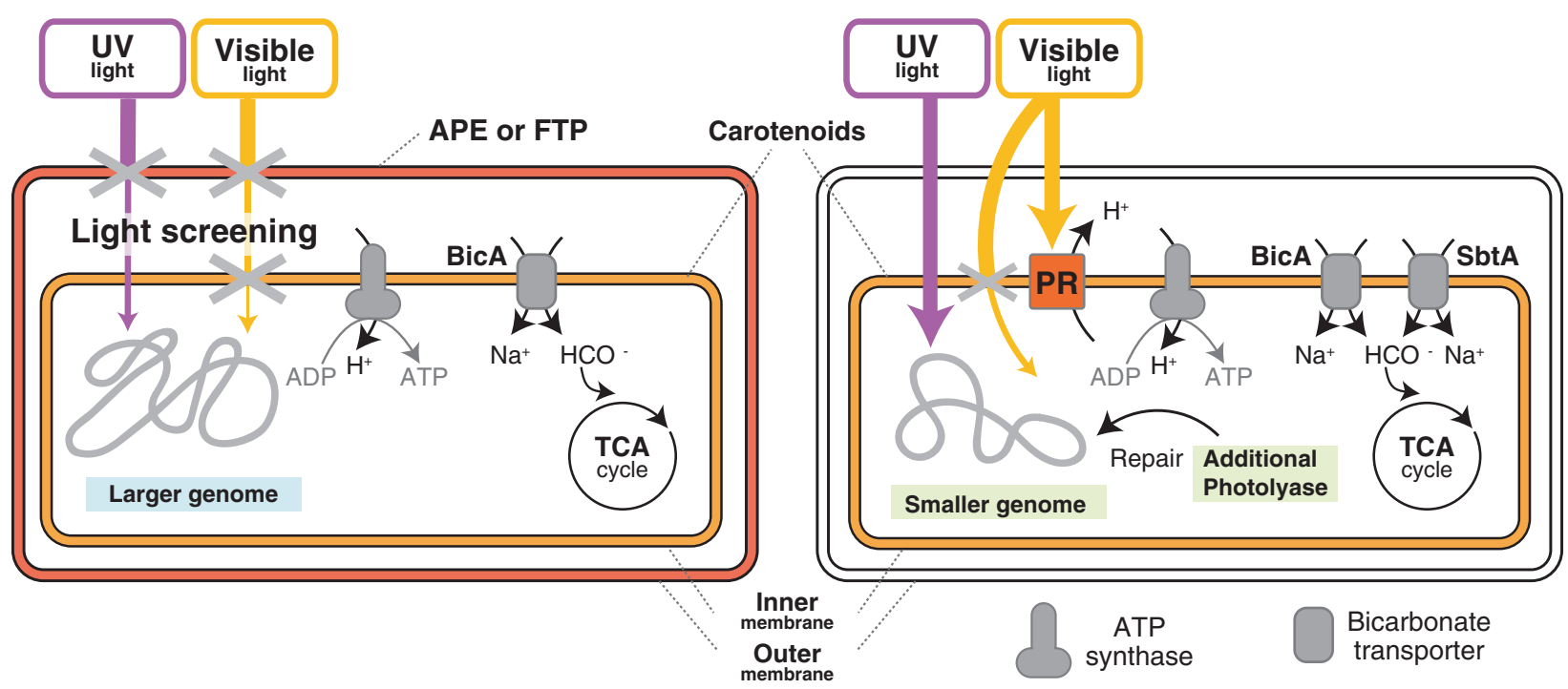

Fig. 7 Schematic figure of the adaptive strategies of PR - and PR+ Flavobacteriia. The background colours in light blue and green represent characteristics of the $\mathrm{PR}-$ and $\mathrm{PR}+$ marine Flavobacteriia, respectively. PR - strains have APEs or FTPs in the outer membrane to block UV and visible light. On the other hand, PR+ strains have neither APEs nor FTPs, but their PR can utilize visible light in the inner membrane. UV light that reaches the DNA in PR+ strains causes DNA damage, which is repaired by photolyases but leads to the smaller genome size of the PR + strains. One gene that is involved in anaplerotic inorganic carbon fixation $(s b t A)$ is biased in $\mathrm{PR}+$ genomes. The PR - strains show signs of adaptation to anaerobic conditions

concentrations and that nitrous oxide reductase genes are more abundant in particle-associated microbial communities than in free-living communities [67]. Thus, the interiors of macroscopic organic aggregates (also known as marine snows) in the upper ocean, which are known to be inhabited by Flavobacteriia [43, 68], are an environment where facultative anaerobic PR - microbes may predominate because their microaerobic (and nutrient-rich) conditions likely decrease the advantage of possessing PR [69]. Although the sampling sites of the strains analyzed in this study were not geographically comprehensive and did not show any geographic trend per the presence and absence of PR genes (Supplementary Fig. S1), we hypothesize that a possible geographic niche of facultative anaerobic PRFlavobacteriia with UV protective pigments might be the surface layer in the eastern tropical north Pacific ocean, whose oxygen concentration is $<10 \mu \mathrm{M}$ even in the nearsurface layer [70]. We envision that large-scale shotgun metagenomic analyses of macroscopic organic aggregates will be required to clarify this hypothesis.

Acknowledgements We thank Koji Hamasaki, Rei Narikawa, Daisuke Nakane, Motomu Matsui, Satoshi Hiraoka, Hiroshi Kiyota, Minoru Ijichi, and Masumi Hasegawa for providing helpful suggestions and assisting in experiments. We are grateful to the captain and crews of R/ V Mirai (JAMSTEC) and R/V Tansei Maru (Atmosphere and Ocean Research Institute, The University of Tokyo and JAMSTEC) for their assistance and support in sample collection. This work was supported by the Japan Science and Technology Agency (CREST), the Japan Society for the Promotion of Science (grant numbers $15 \mathrm{H} 02800$, $15 \mathrm{~K} 14601,15 \mathrm{H} 01725,16 \mathrm{H} 06154,17 \mathrm{H} 05834$, and 15J08516), the Ministry of Education, Culture, Sports, Science, and Technology in 
Table 1 Numbers of genomes that code rhodopsin and APE synthesis genes

\begin{tabular}{llllll}
\hline Taxonomic group & \multicolumn{2}{l}{ Rhodopsin +} & & \multicolumn{2}{l}{ Rhodopsin- } \\
\cline { 2 - 3 } & APE + & APE - & & APE + & APE - \\
\hline Bacteroidetes & 0 & 15 & & 33 & 125 \\
Chloroflexi & 0 & 1 & & 0 & 10 \\
Cyanobacteria & 0 & 1 & & 60 \\
Deinococcusthermus & 0 & 3 & & 0 & 13 \\
Firmicutes & 0 & 2 & & 690 \\
Planctomycetes & 0 & 1 & & 6 \\
Alphaproteobacteria & 0 & 12 & & 0 & 278 \\
Betaproteobacteria & 0 & 2 & & 0 & 197 \\
Gammaproteobacteria & 0 & 10 & & 730 \\
Deltaproteobacteria & 0 & 0 & & 4 & 51 \\
Sphingobacteriia & 0 & 1 & 2 & 7 \\
\hline
\end{tabular}

Genomes that code $>50 \%$ of APE synthesis genes (eggNOG ID: 05C84, 05EWP, 05F6T, 05IAS, 05VME, 0636E, 07T6G, 08M6I, 05DZS, 05EYS, 05H6H, 05M49, 05YNY, 07T3I, 08H1G, and 08XF1) were regarded as APE-synthesizing (APE+) strains. The data were obtained from the eggNOG database version 4.5 [25]

Japan (221S0002 and 16H06279), and the Canon Foundation. Some computations were performed on the NIG supercomputer at ROIS National Institute of Genetics.

Author contributions SY isolated and provided bacterial strains. SY and YN cultivated strains and extracted DNA. KO, MH, YO, and TH sequenced and assembled genomes. YK conducted bioinformatic analyses. YK, MW, and MI conducted knockout experiments. SY, $\mathrm{KK}$, and WI proposed and supervised the project. YK, SY, and WI wrote the manuscript with help from TF, KK, and EFD.

\section{Compliance with ethical standards}

Conflict of interest The authors declare that they have no conflict of interest.

Open Access This article is licensed under a Creative Commons Attribution 4.0 International License, which permits use, sharing, adaptation, distribution and reproduction in any medium or format, as long as you give appropriate credit to the original author(s) and the source, provide a link to the Creative Commons license, and indicate if changes were made. The images or other third party material in this article are included in the article's Creative Commons license, unless indicated otherwise in a credit line to the material. If material is not included in the article's Creative Commons license and your intended use is not permitted by statutory regulation or exceeds the permitted use, you will need to obtain permission directly from the copyright holder. To view a copy of this license, visit http://creativecommons. org/licenses/by/4.0/.

\section{References}

1. Béja O, Aravind L, Koonin EV, Suzuki MT, Hadd A, Nguyen LP, et al. Bacterial rhodopsin: evidence for a new type of phototrophy in the sea. Science. 2000;289:1902-6.

2. Johnson ET, Baron DB, Naranjo B, Bond DR, Schmidt-Dannert C, Gralnick JA. Enhancement of survival and electricity production in an engineered bacterium by light-driven proton pumping. Appl Environ Microbiol. 2010;76:4123-9.

3. Yoshizawa S, Kawanabe A, Ito H, Kandori H, Kogure K. Diversity and functional analysis of proteorhodopsin in marine Flavobacteria. Environ Microbiol. 2012;14:1240-8.

4. Morris RM, Nunn BL, Frazar C, Goodlett DR, Ting YS, Rocap G. Comparative metaproteomics reveals ocean-scale shifts in microbial nutrient utilization and energy transduction. ISME J. 2010;4:673-85.

5. Gómez-Consarnau L, González JM, Coll-Lladó M, Gourdon P, Pascher T, Neutze R, et al. Light stimulates growth of proteorhodopsin-containing marine Flavobacteria. Nature. 2007;445:210-3.

6. González JM, Fernández-Gómez B, Fernàndez-Guerra A, GómezConsarnau L, Sánchez O, Coll-Lladó M, et al. Genome analysis of the proteorhodopsin-containing marine bacterium Polaribacter sp. MED152 (Flavobacteria). Proc Natl Acad Sci USA. 2008;105:8724-9.

7. Palovaara J, Akram N, Baltar F, Bunse C, Forsberg J, Pedrós-Alió $\mathrm{C}$, et al. Stimulation of growth by proteorhodopsin phototrophy involves regulation of central metabolic pathways in marine planktonic bacteria. Proc Natl Acad Sci USA. 2014;111: E3650-58.

8. Dubinsky V, Haber M, Burgsdorf I, Saurav K, Lehahn Y, Malik A, et al. Metagenomic analysis reveals unusually high incidence of proteorhodopsin genes in the ultraoligotrophic Eastern Mediterranean Sea. Environ Microbiol. 2017;19:1077-90.

9. Ottesen EA, Young CR, Gifford SM, Eppley JM, Marin R, Schuster SC, et al. Multispecies diel transcriptional oscillations in open ocean heterotrophic bacterial assemblages. Science. 2014;345:207-12.

10. Cordero OX, Ventouras L-A, DeLong EF, Polz MF. Public good dynamics drive evolution of iron acquisition strategies in natural bacterioplankton populations. Proc Natl Acad Sci USA. 2012;109:20059-64.

11. Fernández-Gomez B, Richter $\mathrm{M}$, Schüler M, Pinhassi J, Acinas SG, González JM, et al. Ecology of marine Bacteroidetes: a comparative genomics approach. ISME J. 2013;7:1026-37.

12. Lauro FM, McDougald D, Thomas T, Williams TJ, Egan S, Rice $\mathrm{S}$, et al. The genomic basis of trophic strategy in marine bacteria. Proc Natl Acad Sci USA. 2009;106:15527-33.

13. Thrash JC, Temperton B, Swan BK, Landry ZC, Woyke T, DeLong EF, et al. Single-cell enabled comparative genomics of a deep ocean SAR11 bathytype. ISME J. 2014;8:1440-51.

14. Jothi R, Przytycka TM, Aravind L. Discovering functional linkages and uncharacterized cellular pathways using phylogenetic profile comparisons: a comprehensive assessment. BMC Bioinform. 2007;8:173.

15. Škunca N, Dessimoz C. Phylogenetic profiling: how much input data is enough? PLoS ONE. 2015;10:e0114701.

16. Neumann B, Pospiech A, Schairer HU. Rapid isolation of genomic DNA from gram-negative bacteria. Trends Genet. 1992;8:332-3.

17. Pruitt KD, Tatusova T, Maglott DR. NCBI reference sequence (RefSeq): a curated non-redundant sequence database of genomes, transcripts and proteins. Nucleic Acids Res. 2005;33:D501-4.

18. The UniProt Consortium. UniProt: a hub for protein information. Nucleic Acids Res. 2014;43:D204-12.

19. Simão FA, Waterhouse RM, Ioannidis $P$, Kriventseva EV, Zdobnov EM. BUSCO: assessing genome assembly and annotation completeness with single-copy orthologs. Bioinformatics. 2015;31:3210-2.

20. Mongodin EF, Nelson K, Daugherty S, Deboy R, Wister J, Khouri $\mathrm{H}$, et al. The genome of Salinibacter ruber: convergence and gene 
exchange among hyperhalophilic bacteria and archaea. Proc Natl Acad Sci USA. 2005; 102:18147-52.

21. Lagesen K, Hallin P, Rødland EA, Stærfeldt H-H, Rognes T, Ussery DW. RNAmmer: consistent and rapid annotation of ribosomal RNA genes. Nucleic Acids Res. 2007;35:3100-8.

22. Lowe TM, Eddy SR. tRNAscan-SE: a program for improved detection of transfer RNA genes in genomic sequence. Nucleic Acids Res. 1997;25:955-64.

23. Hyatt D, Chen G-L, LoCascio PF, Land ML, Larimer FW, Hauser LJ. Prodigal: prokaryotic gene recognition and translation initiation site identification. BMC Bioinform. 2010;11:119.

24. Huerta-Cepas J, Forslund K, Pedro Coelho L, Szklarczyk D, Juhl Jensen $\mathrm{L}$, von Mering $\mathrm{C}$, et al. Fast genome-wide functional annotation through orthology assignment by eggNOG-mapper. Mol Biol Evol. 2017;34:2115-22.

25. Huerta-Cepas J, Szklarczyk D, Forslund K, Cook H, Heller D, Walter MC, et al. eggNOG 4.5: a hierarchical orthology framework with improved functional annotations for eukaryotic, prokaryotic and viral sequences. Nucleic Acids Res. 2015;44: D286-93.

26. Katoh K, Misawa $\mathrm{K}$, Kuma Ki, Miyata T. MAFFT: a novel method for rapid multiple sequence alignment based on fast Fourier transform. Nucleic Acids Res. 2002;30:3059-66.

27. Capella-Gutiérrez S, Silla-Martínez JM, Gabaldón T. trimAl: a tool for automated alignment trimming in large-scale phylogenetic analyses. Bioinformatics. 2009;25:1972-3.

28. Darriba D, Taboada GL, Doallo R, Posada D. ProtTest 3: fast selection of best-fit models of protein evolution. Bioinformatics. 2011;27:1164-5.

29. Stamatakis A. RAxML-VI-HPC: maximum likelihood-based phylogenetic analyses with thousands of taxa and mixed models. Bioinformatics. 2006;22:2688-90.

30. Yoshizawa S, Kumagai Y, Kim H, Ogura Y, Hayashi T, Iwasaki $\mathrm{W}$, et al. Functional characterization of flavobacteria rhodopsins reveals a unique class of light-driven chloride pump in bacteria. Proc Natl Acad Sci USA. 2014;111:6732-7.

31. Brunner E, Munzel U. The nonparametric Behrens-Fisher problem: asymptotic theory and a small-sample approximation. Biom J. 2000;42:17-25.

32. Storey JD, Tibshirani R. Statistical significance for genomewide studies. Proc Natl Acad Sci USA. 2003;100:9440-5.

33. Nakamura Y, Kaneko T, Hirosawa M, Miyajima N, Tabata S. CyanoBase, a www database containing the complete nucleotide sequence of the genome of Synechocystis sp. strain PCC6803. Nucleic Acids Res. 1998;26:63-67.

34. Stanier R, Kunisawa R, Mandel M, Cohen-Bazire G. Purification and properties of unicellular blue-green algae (order Chroococcales). Bacteriol Rev. 1971;35:171-205.

35. Marchler-Bauer A, Derbyshire MK, Gonzales NR, Lu S, Chitsaz F, Geer LY, et al. CDD: NCBI's conserved domain database. Nucleic Acids Res. 2014;43:D222-6.

36. Sunagawa S, Coelho LP, Chaffron S, Kultima JR, Labadie K, Salazar G, et al. Structure and function of the global ocean microbiome. Science. 2015;348:1261359.

37. Uchiyama I. MBGD: microbial genome database for comparative analysis. Nucleic Acids Res. 2003;31:58-62.

38. Yoshihara S, Suzuki F, Fujita H, Geng XX, Ikeuchi M. Novel putative photoreceptor and regulatory genes required for the positive phototactic movement of the unicellular motile cyanobacterium Synechocystis sp. PCC 6803. Plant Cell Physiol. 2000;41:1299-304.

39. Bhaya D. Light matters: phototaxis and signal transduction in unicellular cyanobacteria. Mol Microbiol. 2004;53:745-54.

40. Yoshihara S, Ikeuchi M. Phototactic motility in the unicellular cyanobacterium Synechocystis sp. PCC 6803. Photoch Photobio Sci. 2004;3:512-8.
41. Huynen MA, Snel B, von Mering C, Bork P. Function prediction and protein networks. Curr Opin Cell Biol. 2003;15:191-8.

42. Pinhassi J, DeLong EF, Béjà O, González JM, Pedrós-Alió C. Marine bacterial and archaeal ion-pumping rhodopsins: genetic diversity, physiology, and ecology. Microbiol Mol Biol Rev. 2016;80:929-54.

43. Kirchman DL. The ecology of Cytophaga-Flavobacteria in aquatic environments. FEMS Microbiol Ecol. 2002;39:91-100.

44. Chan Y-K, McCormic WA, Watson RJ. A new nos gene downstream from nosDFY is essential for dissimilatory reduction of nitrous oxide by Rhizobium (Sinorhizobium) meliloti. Microbiology. 1997;143:2817-24.

45. Coyle CL, Zumft WG, Kroneck PM, Korner H, Jakob W. Nitrous oxide reductase from denitrifying Pseudomonas perfectomarina. Purification and properties of a novel multicopper enzyme. FEBS J. 1985;153:459-67.

46. Bauer M, Kube M, Teeling H, Richter M, Lombardot T, Allers E, et al. Whole genome analysis of the marine Bacteroidetes 'Gramella forsetii'reveals adaptations to degradation of polymeric organic matter. Environ Microbiol. 2006;8:2201-13.

47. Nordlund P, Reichard P. Ribonucleotide reductases. Annu Rev Biochem. 2006;75:681-6.

48. Schellhorn HE, Hassan HM. Transcriptional regulation of katE in Escherichia coli K-12. J Bacteriol. 1988;170:4286-92.

49. Imlay JA. The molecular mechanisms and physiological consequences of oxidative stress: lessons from a model bacterium. Nat Rev Microbiol. 2013;11:443-54 .

50. Mishra S, Imlay JA. An anaerobic bacterium, Bacteroides thetaiotaomicron, uses a consortium of enzymes to scavenge hydrogen peroxide. Mol Microbiol. 2013;90:1356-71.

51. Preisig O, Anthamatten D, Hennecke H. Genes for a microaerobically induced oxidase complex in Bradyrhizobium japonicum are essential for a nitrogen-fixing endosymbiosis. Proc Natl Acad Sci USA. 1993;90:3309-13.

52. González JM, Pinhassi J, Fernández-Gómez B, Coll-Lladó M, González-Velázquez $\mathrm{M}$, Puigbò $\mathrm{P}$, et al. Genomics of the proteorhodopsin-containing marine flavobacterium Dokdonia sp. strain MED134. Appl Environ Microbiol. 2011;77:8676-86.

53. Cimermancic P, Medema MH, Claesen J, Kurita K, Brown LCW, Mavrommatis $\mathrm{K}$, et al. Insights into secondary metabolism from a global analysis of prokaryotic biosynthetic gene clusters. Cell. 2014;158:412-21.

54. Goel AK, Rajagopal L, Nagesh N, Sonti RV. Genetic locus encoding functions involved in biosynthesis and outer membrane localization of xanthomonadin in Xanthomonas oryzae pv. oryzae. J Bacteriol. 2002;184:3539-48.

55. Wang Y, Qian G, Li Y, Wang Y, Wang Y, Wright S, et al. Biosynthetic mechanism for sunscreens of the biocontrol agent Lysobacter enzymogenes. PLoS ONE. 2013;8:e66633.

56. Irschik $H$, Reichenbach $H$. Intracellular location of flexirubins in Flexibacter elegans (Cytophagales). BBA-Biomembr. 1978;510:1-10.

57. Gao Q, Garcia-Pichel F. Microbial ultraviolet sunscreens. Nat Rev Microbiol. 2011;9:791-802.

58. Fautz E, Reichenbach H. A simple test for flexirubin-type pigments. FEMS Microbiol Lett. 1980;8:87-91.

59. Reichenbach H, Kohl W, Böttger-Vetter A, Achenbach H. Flexirubin-type pigments in Flavobacterium. Arch Microbiol. 1980;126:291-3.

60. Venil CK, Zakaria ZA, Usha R, Ahmad WA. Isolation and characterization of flexirubin type pigment from Chryseobacterium sp. UTM-3 T. Biocatal Agric Biotechnol. 2014;3:103-7.

61. Nedashkovskaya OI, Kim SB, Lysenko AM, Frolova GM, Mikhailov VV, Lee KH, et al. Description of Aquimarina muelleri gen. nov., sp. nov., and proposal of the reclassification of 
[Cytophaga] latercula Lewin 1969 as Stanierella latercula gen. nov., comb. nov. Int J Syst Evol Microbiol. 2005;55:225-9.

62. Sancar A. Structure and function of DNA photolyase. Biochemistry. 1994;33:2-9.

63. Brash DE, Haseltine WA. UV-induced mutation hotspots occur at DNA damage hotspots. Nature. 1982;298:189-92.

64. Viklund J, Ettema TJ, Andersson SG. Independent genome reduction and phylogenetic reclassification of the oceanic SAR11 clade. Mol Biol Evol. 2011;29:599-15.

65. Kim Y-S, Kim N-H, Yeom S-J, Kim S-W, Oh D-K. In vitro characterization of a recombinant Blh protein from an uncultured marine bacterium as a $\beta$-carotene $15,15^{\prime}$-dioxygenase. J Biol Chem. 2009;284:15781-93.

66. Bruns A, Rohde M, Berthe-Corti L. Muricauda ruestringensis gen. nov., sp. nov., a facultatively anaerobic, appendaged bacterium from German North Sea intertidal sediment. Int J Syst Evol Microbiol. 2001;51:1997-6.
67. Ganesh S, Parris DJ, DeLong EF, Stewart FJ. Metagenomic analysis of size-fractionated picoplankton in a marine oxygen minimum zone. ISME J. 2014;8:187-211.

68. Buchan A, LeCleir GR, Gulvik CA, González JM. Master recyclers: features and functions of bacteria associated with phytoplankton blooms. Nat Rev Microbiol. 2014;12:686-98.

69. Ploug H, Kühl M, Buchholz-Cleven B, Jørgensen BB. Anoxic aggregates-an ephemeral phenomenon in the pelagic environment? Aquat Microb Ecol. 1997;13:285-94.

70. Paulmier A, Ruiz-Pino D. Oxygen minimum zones (OMZs) in the modern ocean. Prog Oceanogr. 2009;80:113-28.

71. Letunic I, Bork P. Interactive tree of life (iTOL)v3: an online tool for the display and annotation of phylogenetic and other trees. Nucleic Acids Res. 2016;44:W242-5.

72. Brown NP, Leroy C, Sander C. MView: a web-compatible database search or multiple alignment viewer. Bioinformatics. 1998;14:380-1. 